\title{
OPEN Deep learning model for classification and bioactivity prediction of essential oil-producing plants from Egypt
}

\author{
Noha E. El-Attar ${ }^{1 \bowtie}$, Mohamed K. Hassan², Othman A. Alghamdi ${ }^{3}$ \& Wael A. Awad ${ }^{4,5}$
}

Reliance on deep learning techniques has become an important trend in several science domains including biological science, due to its proven efficiency in manipulating big data that are often characterized by their non-linear processes and complicated relationships. In this study, Convolutional Neural Networks (CNN) has been recruited, as one of the deep learning techniques, to be used in classifying and predicting the biological activities of the essential oil-producing plant/s through their chemical compositions. The model is established based on the available chemical composition's information of a set of endemic Egyptian plants and their biological activities. Another type of machine learning algorithms, Multiclass Neural Network (MNN), has been applied on the same Essential Oils (EO) dataset. This aims to fairly evaluate the performance of the proposed CNN model. The recorded accuracy in the testing process for both CNN and MNN is $98.13 \%$ and $81.88 \%$, respectively. Finally, the $\mathrm{CNN}$ technique has been adopted as a reliable model for classifying and predicting the bioactivities of the Egyptian EO-containing plants. The overall accuracy for the final prediction process is reported as approximately $97 \%$. Hereby, the proposed deep learning model could be utilized as an efficient model in predicting the bioactivities of, at least Egyptian, EOs-producing plants.

Recently, Artificial Intelligence (AI) has become one of the vigorous science that infiltrated a huge number of modern life issues such as chemical engineering, water treatment, and biological domain like genomic and proteomic studies which are especially characterized by complicated and non-linear processes ${ }^{1}$. Deep learning is one of the most promising branches of artificial intelligence with proven power in taking the raw features extracted from the extremely large data sets, such as the data produced from genomics, chemistry, and pharmaceutical laboratories. Processing these data result in inferred patterns and training process-based predictive models ${ }^{2}$.

Essential Oils (EOs) are biologically effective organic compounds extracted from different parts of the aromatic plants such as flowers, leaves, and barks to name a few ${ }^{3}$. Due to their wide range of biological activities, these natural products are widely used in complementary and alternative medicine (CAM). Replacing the inorganic chemistry by natural alternatives is still hot topic in recent biological area of research. This is because the inorganic chemical products may bereave harmful influences when used in health-related industries, such as medicine, pharmaceutics, cosmetics, food, and beverages. Therefore, the modern researches head to find the alternative natural products, including EOs, due to their greater ability to adapt to alive organs of the human body with, sometimes, limited side effects ${ }^{4}$.

Generally, the bioactivity of the EO-producing plant depends on the chemical structure and EOs content, which determine the overall bioactivity of such plant. EOs are, in fact, composed of different combinations of low nuclear weight natural blends with complete organic locomotion. According to their structure, these dynamic blends can be categorized into some significant pools (e.g. hydrocarbons, oxygenated mixtures, and sulfur or conceivably nitrogen). These mixes' pools are the mystery key for the biological activity of each $\mathrm{EO}^{3}$.

\footnotetext{
${ }^{1}$ Information Systems Department, Faculty of Computers and Artificial Intelligence, Banha University, Banha, Egypt. ${ }^{2 B i o t e c h n o l o g y ~ P r o g r a m, ~ Z o o l o g y ~ D e p a r t m e n t, ~ F a c u l t y ~ o f ~ S c i e n c e, ~ P o r t ~ S a i d ~ U n i v e r s i t y, ~ P o r t ~ S a i d, ~}$ Egypt. ${ }^{3}$ Department of Biological Sciences, Collage of Science, University of Jeddah, Jeddah, Kingdom of Saudi Arabia. ${ }^{4}$ Mathematics and Computer Science Department, Faculty of Science, Port Said University, Port Said, Egypt. ${ }^{5}$ Computer Science Department, Faculty of Computers and Information, Damietta University, Damietta, Egypt. ${ }^{\varpi}$ email: noha.ezzat@fci.bu.edu.eg
} 


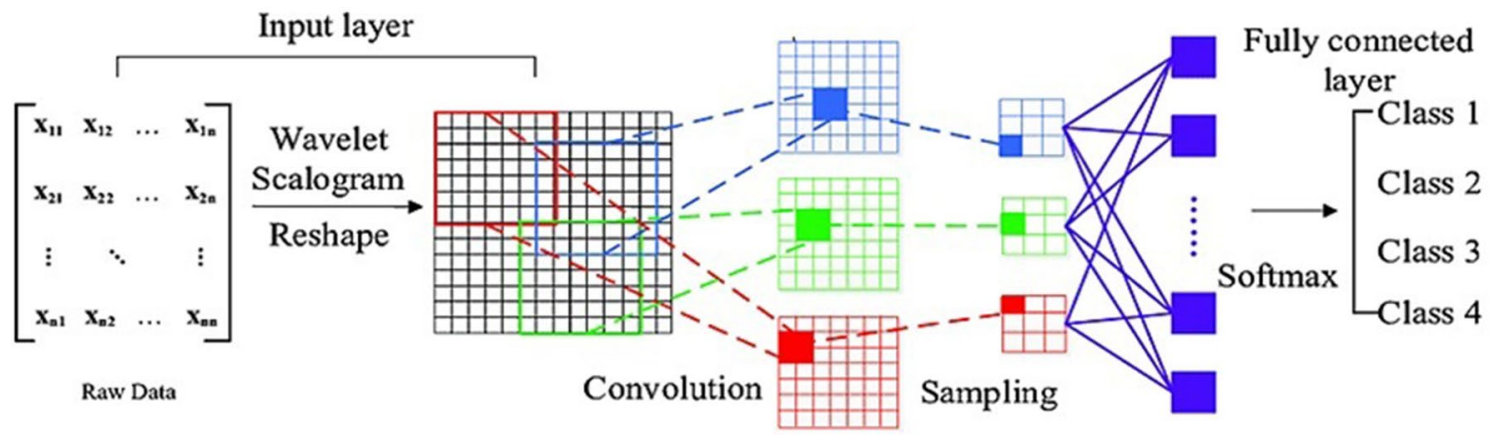

Figure 1. Standard Convolutional Neural Network Architecture usually consists of an input layer, convolution layer, Max pooling and the final fully-connected neural network layer which gives the output based on the activation function ${ }^{9}$.

The biological activities of the EOs may include antiseptics, antimicrobials, antifungals, antioxidant, antitumor, antivirals, and/or anti-inflammatories. Moreover, these activities vary according to the chemical constitution, which may differ from plant to another according to their geographic location, agriculture conditions, climatic or seasonal changes ${ }^{4}$. Noteworthy, the evaluation of the EOs' bioactivities cannot be constantly credited to one single compound in the EOs mixture. The genuine connections between the EO's cocktail and its biological activities are highly non-linear, especially when considered across variable pools of chemical structures. Hence, reliance on traditional techniques in predicting the biological impact for such activity data with this variety of structures is a troublesome issue $e^{5}$. Therefore, developing a deep learning-based computational model to categorize and predict the biological activities of EOs-producing plants based on their chemical construction's variations, without recourse to in-vitro experiments, could save time and cost.

Machine learning (ML) algorithms, especially Artificial Neural Networks (ANN), have been proposed to contribute in solving several biological issues in the recent decades ${ }^{6}$. ANN, in general, can be depicted as a numerical model of a particular structure, comprising of some of the single processing components (i.e. nodes and neurons), constructed between inter-connected layers. Each entire layer is mainly composed of hidden neurons which are responsible for transforming the input values and sending the outputs to the other associated neurons ${ }^{1}$. Recently, due to the expansion of the biological information, the fully connected neural network would have a huge number of parameters, which needs full processing inside the network layers to deliver the desired output. Deep learning approaches have proven their efficiency in the applications whose data are characterized by their large quantities, high dimensionality, and highly structured. Thus, deep learning approaches are widely used in image processing due to the nature of the image which contains many thousands of variables (pixels) that can be clearly grouped into well- defined objects ${ }^{6}$. However, deep learning approaches are no longer limited to image processing domain, where it is recently considered an attractive solution for some types of text classification such as DNA sequences classification problems ${ }^{7}$. From this standpoint, deep learning can be an efficient learning approach for dealing with the complex composition of the chemical compounds and their interrelationships with biological activities.

One of the efficient models for the deep learning is the Convolutional Neural Network (CNN). The CNN is characterized by two novel types of layers: convolution and pooling layers. These layers are based on using filters to convolve the range of the input data to a smaller range, detecting important or specific parts within this range ${ }^{8}$. The CNN usually consists of Input Layer, Convolution Layer (i.e. produces a matrix of dimension smaller than the input matrix), ReLU or Rectified Linear Unit.ReLU is mathematically expressed as Max $(0, x)$ (i.e. it means that any number below 0 is converted to 0 , while any positive number is allowed to pass as it is), Max pooling (i.e. passes the maximum value from amongst a small collection of elements of the incoming matrix to the output) and the final output layer (i.e. a fully-connected neural network layer, which makes the output based on the activation function), as shown in Fig. 19 .

This study seeks to classify and predict the biological activities of the Egyptian essential oil-producing plants based on their EOs content as an experimental case study. The classification is implemented based on two algorithms: Multiclass Neural Network (MNN) and Convolutional Neural Network (CNN), to evaluate the efficiency of both machine and deep learning techniques. The effective algorithm is adopted in developing a biological activity prediction model EOs-producing cases cultivated in Egypt. The research skills in this study are organized as follow; first, presenting the results obtained from implementing the two algorithms; MNN as a machine learning algorithm and CNN as a deep learning algorithm, when applied on the Egyptian essential oils dataset. Next, these results were discussed and evaluated for the existing dataset and the new untested datasets. Finally the methodologies followed in this work are discussed.

\section{Results}

Using MNN- and CNN-based algorithms to classify the plants' EOs bioactivity. The results recorded from the classification process for both MNN and CNN algorithms are summarized in Tables 1 and 2. In the CNN and MNN algorithms, the training processes show an overall accuracy of $100 \%$ and $99.2 \%$, of correct classification of essential oils activity, respectively. Whilst, for the testing stage, the overall accuracy achieved by CNN and $\mathrm{MNN}$ is $98.13 \%$ and $81.88 \%$, respectively. 


\begin{tabular}{|l|l|l|l|l|l|l|l|l|}
\hline \multirow{2}{*}{$\begin{array}{l}\text { Bioactivity class } \\
\text { Classifiers }\end{array}$} & \multicolumn{2}{|l|}{ True positive } & \multicolumn{2}{l|}{ True negative } & \multicolumn{2}{l|}{ False positive } & \multicolumn{2}{l|}{ False negative } \\
\cline { 2 - 10 } & MNN & CNN & MNN & CNN & MNN & CNN & MNN & CNN \\
\hline Antiviral & $50 \%$ & $97.7 \%$ & $94.4 \%$ & $98.2 \%$ & $5.6 \%$ & $1.8 \%$ & $50 \%$ & $2.3 \%$ \\
\hline Antiwormal & $100 \%$ & $100 \%$ & $98.3 \%$ & $98.5 \%$ & $1.7 \%$ & $1.5 \%$ & 0 & $0 \%$ \\
\hline Anti-inflammatory & $90 \%$ & $100 \%$ & $80 \%$ & $100 \%$ & $20 \%$ & $0 \%$ & $10 \%$ & $0 \%$ \\
\hline Anticancer & $85.7 \%$ & $94 \%$ & $96.2 \%$ & $93.3 \%$ & $3.8 \%$ & $6.7 \%$ & $14.3 \%$ & $6 \%$ \\
\hline Antioxidant & $75.9 \%$ & $98.4 \%$ & $80.6 \%$ & $98.6 \%$ & $19.4 \%$ & $1.4 \%$ & $24.1 \%$ & $1.6 \%$ \\
\hline Antimicrobial & $78.8 \%$ & $97.4 \%$ & $66.7 \%$ & $97.3 \%$ & $33.3 \%$ & $2.7 \%$ & $21.2 \%$ & $2.6 \%$ \\
\hline Antifungal & $81.3 \%$ & $98 \%$ & $86.4 \%$ & $99.2 \%$ & $13.6 \%$ & $0.8 \%$ & $18.8 \%$ & $2 \%$ \\
\hline Cytotoxic activity & $58.3 \%$ & $96.6 \%$ & $93.8 \%$ & $98.7 \%$ & $6.3 \%$ & $1.3 \%$ & $41.7 \%$ & $3.4 \%$ \\
\hline
\end{tabular}

Table 1. The confusion matrix values for $\mathrm{MNN}$ and $\mathrm{CNN}$ algorithms in testing stage.

\begin{tabular}{|l|l|l|l|l|l|l|l|l|}
\hline \multirow{2}{*}{$\begin{array}{l}\text { Bioactivity class } \\
\text { Metrics }\end{array}$} & \multicolumn{2}{l}{$\begin{array}{l}\text { Average } \\
\text { accuracy }\end{array}$} & \multicolumn{2}{l}{ Precision } & \multicolumn{2}{l|}{ Recall } & \multicolumn{2}{l|}{ F1 score } \\
\cline { 2 - 10 } & MNN & CNN & MNN & CNN & MNN & CNN & MNN & CNN \\
\hline Antiviral & 0.72 & 0.99 & 0.9 & 0.98 & 0.5 & 0.99 & 0.64 & 0.99 \\
\hline Antiwormal & 0.99 & 0.99 & 0.98 & 0.99 & 1 & 1 & 0.99 & 0.99 \\
\hline Anti-inflammatory & 0.85 & 1 & 0.82 & 1 & 0.9 & 1 & 0.86 & 1 \\
\hline Anticancer & 0.9 & 0.94 & 0.96 & 0.93 & 0.86 & 0.94 & 0.9 & 0.94 \\
\hline Antioxidant & 0.78 & 0.99 & 0.8 & 0.99 & 0.76 & 0.98 & 0.78 & 0.98 \\
\hline Antimicrobial & 0.73 & 0.97 & 0.7 & 0.97 & 0.79 & 0.97 & 0.74 & 0.97 \\
\hline Antifungal & 0.84 & 0.99 & 0.86 & 0.99 & 0.812 & 0.98 & 0.83 & 0.99 \\
\hline Cytotoxic activity & 0.76 & 0.98 & 0.9 & 0.99 & 0.58 & 0.97 & 0.71 & 0.98 \\
\hline
\end{tabular}

Table 2. The accuracy and relevance metrics for MNN and CNN algorithms in testing stage.

\begin{tabular}{|l|l|l|l|l|}
\hline $\begin{array}{l}\text { Bioactivity class } \\
\text { Classifiers }\end{array}$ & True positive & True negative & False positive & False negative \\
\hline Antiviral & $98 \%$ & $96.3 \%$ & $3.7 \%$ & $2 \%$ \\
\hline Antiwormal & $100 \%$ & $98.3 \%$ & $1.7 \%$ & $0 \%$ \\
\hline Anti-inflammatory & $100 \%$ & $100 \%$ & $0 \%$ & $0 \%$ \\
\hline Anticancer & $97 \%$ & $94.7 \%$ & $5.3 \%$ & $3 \%$ \\
\hline Antioxidant & $100 \%$ & $95 \%$ & $5 \%$ & $0 \%$ \\
\hline Antimicrobial & $94 \%$ & $93.5 \%$ & $6.5 \%$ & $6 \%$ \\
\hline Antifungal & $100 \%$ & $96.6 \%$ & $3.4 \%$ & $0 \%$ \\
\hline Cytotoxic activity & $94 \%$ & $93.1 \%$ & $6.9 \%$ & $6 \%$ \\
\hline
\end{tabular}

Table 3. The confusion matrix values for the predicting model.

The confusion matrix is the most suitable way to validate the classification performance. Here, the confusion matrix of the MNN and CNN classification is presented in Table 1. It consists of four outcomes of binary classifiers: True Positive, False Positive, True Negative, and False Negative. Also, accuracy, precision, recall, and F1 score are different metrics that are used for evaluating the classification efficiency based on the values of the confusion matrix ${ }^{10}$. The four metrics for the bioactivities classes have been calculated and documented in Table 2 .

Using a CNN-based algorithms to build a prediction model for the EOs bioactivity. In accordance with the completion of the classification process, the CNN algorithm show high accuracy for the training and testing processes. This is due to its capabilities in dealing with the huge number of data and focusing on the high impact features in the dataset. Thus, the biological activity prediction model has been built based on the CNN proposed algorithm. The overall accuracy for predicting the biological activities for previously unknown Egyptian essential oils components has been recorded as approximately $97 \%$. The concluded metrics values and the discrimination outcomes of the proposed CNN prediction model are reported in Tables 3 and 4, and in Fig. 2. 


\begin{tabular}{|l|l|l|l|l|}
\hline $\begin{array}{l}\text { Bioactivity class } \\
\text { Metrics }\end{array}$ & Average accuracy & Precision & Recall & F1 score \\
\hline Antiviral & 0.97 & 0.96 & 0.98 & 0.97 \\
\hline Antiwormal & 0.99 & 0.98 & 1 & 0.99 \\
\hline Anti-inflammatory & 1 & 1 & 1 & 1 \\
\hline Anticancer & 0.96 & 0.95 & 0.97 & 0.96 \\
\hline Antioxidant & 0.98 & 0.95 & 1 & 0.98 \\
\hline Antimicrobial & 0.94 & 0.94 & 0.94 & 0.94 \\
\hline Antifungal & 0.98 & 0.97 & 1 & 0.98 \\
\hline Cytotoxic activity & 0.94 & 0.93 & 0.94 & 0.94 \\
\hline
\end{tabular}

Table 4. The accuracy and relevance metrics for the predicting model.

\section{Discussion}

Adopting the idea of using AI, especially machine learning and deep learning algorithms, has become a vital topic in medical and biological problem solving. EOs are considered as one of the well-known natural products which have medical-relevant use and biological-defense activities against several types of viruses, bacteria, and cancer ${ }^{11}$. However, the lack of the complete information about their intrinsic chemical variability and their function makes it difficult to confirm its consistent activities ${ }^{4}$.

Many researchers have adopted the AI in EO's researches aiming to reduce the in-vitro procedures and to make reasonable predications of the experiment results. For instance, Ragno et al. (2020) have developed an unsupervised machine learning algorithm to cluster the EOs and to identify the EOs that have strong ability in inhibiting bacterial growth of all bacterial strains ${ }^{12}$. Moreover, Artini et al. (2018) have exhibited a binary classification model based on ML to classify the essential oils activities from different Mediterranean plants against pseudomonas aeruginosa ${ }^{13}$. Similarly, Daynac et al. (2015) have used the fast artificial neural networks (FNN) to predict the antimicrobial activity of 49 EOs against four types of pathogens. The FNN algorithm predicted more than $70 \%$ of the antimicrobial activities within a $10 \mathrm{~mm}$ maximum error range $\mathrm{e}^{4}$.

Egypt is one of the biggest countries in the world in exporting the high-quality raw material of more than 150 medicinal and aromatic plants. The variety and massiveness of aromatic plant species in Egypt, stemming from the climatic conditions in its environment, may stimulate the accumulation of high concentrated secondary metabolites. This makes the Egyptian aromatic plants are considered between the most promising sources for many biologically active compounds ${ }^{14}$. Thus, there is an imperious need to extend the scientific knowledge base of the aromatic species in Egypt by the modern AI methodologies using machine and deep learning.

The experimental study, here, is applied on the Egyptian case for the essential oils. The data were collected from several peer researches article including in-vitro experiments. For each case, the in-vitro experiment may only focus in analyzing the chemical composition of the EO, or apply one or more of EOs on a specific infection type (e.g. bacteria, viruses, or cancer). This encouraged the authors, here, to experiment the machine and deep learning algorithms in predicting the biological activities for some of the Egyptian essential oils-producing plants. The training and testing processes are conducted on a novel dataset of EOs-producing plants from Egypt, that are collected and manipulated by authors from peer reviewed scientific researches ${ }^{11-55}$. The resulting dataset consists of a sample of one hundred and twenty (120) plants. The total chemical compounds extracted from this dataset are 573 compounds. In the proposed experimental case, the biological activities of the essential oils are classified according to eight categories of bio-activities (anticancer, antioxidant, antimicrobial, antifungal, antiviral, anti-wormal, anti-inflammatory, and cytotoxic activities).

In order to classify the EO's according to their biological activities, two classification models rely on the supervised learning are constructed; the first one is based on the Multiclass Neural Network (MNN) (i.e. the Multiclass N.N module in Azure). The second model depends on the Convolutional Neural Network (CNN) and it has been implemented by Python and executed as a module on the open source Azure Machine learning studio ${ }^{56}$. Figure 3 presents an inclusive flowchart for the proposed methodology stages. The two proposed supervised learning algorithms are applied on nearly 68,760 values that represent the percentage of chemical compounds concentrations in the EO's dataset on study. The training process for both MNN and CNN algorithms is done on $60 \%$ of the dataset, whilst the remaining $40 \%$ is divided between the testing and the prediction processes. That means the actual size for the training, testing, and validation processes are 45,840, 11,460, and 11,460 values, respectively. Figure 4 shows a sample for the EOs dataset stored in azure format.

In the proposed $\mathrm{MNN}$ algorithm, the training model runs a sequence of binary classifiers and trains each to decide a separate classification outcome according to the softmax activation function results. The architecture of the proposed MNN is a fully connected layers network with one hidden layer contains 50 nodes, and an output layer with 8 nodes each one represents an output class. The outputs of the hidden layer $O h_{i}$ and the output layer $O_{k}$ are calculated by Eq. 1 and 3 respectively ${ }^{57}$.

$$
O h_{i}=\frac{1}{1+e^{-z h_{i}}} \forall i=1, \ldots \ldots, 50
$$

where 


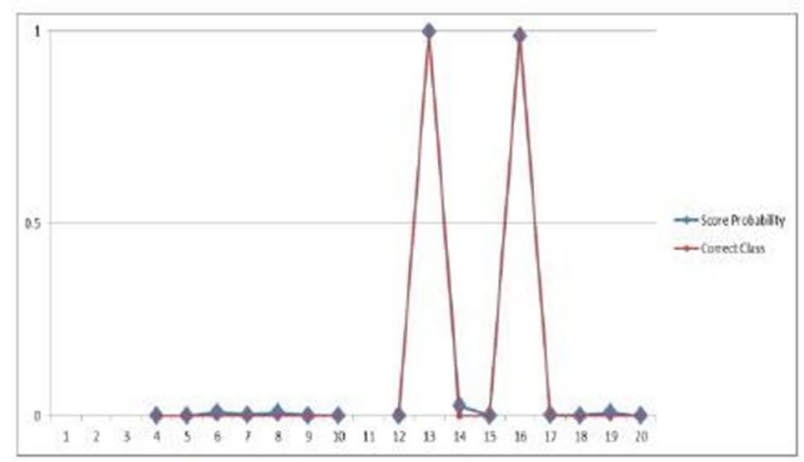

Score Probabilities for Anticancer Activity

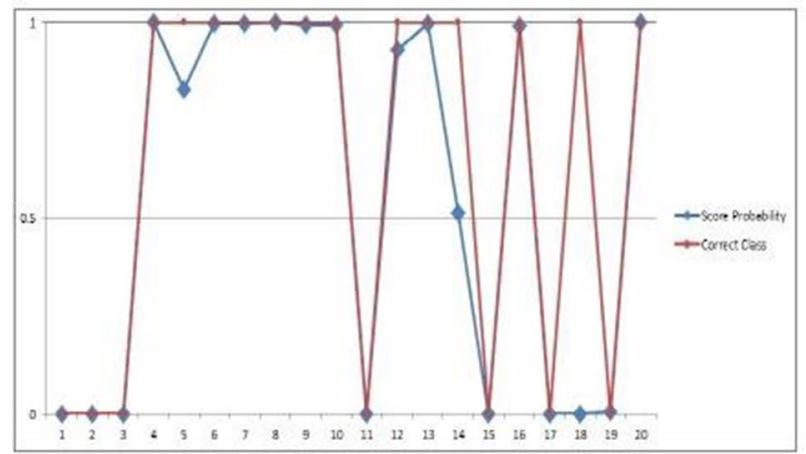

Score Probabilities for Antioxident Activity

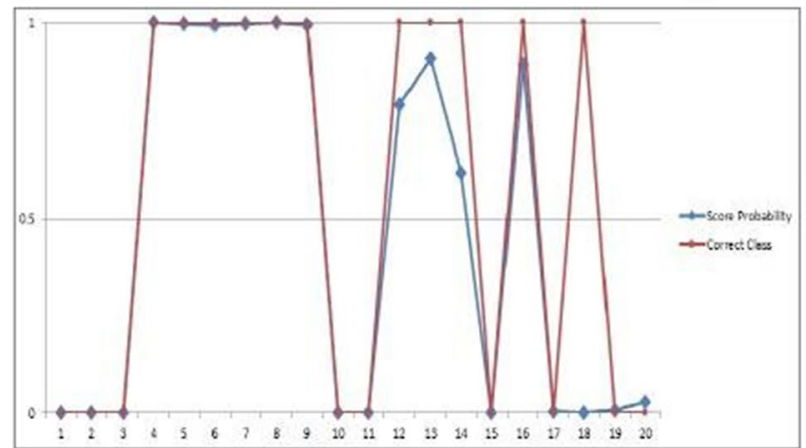

Score Probabilities for Antifungal Activity

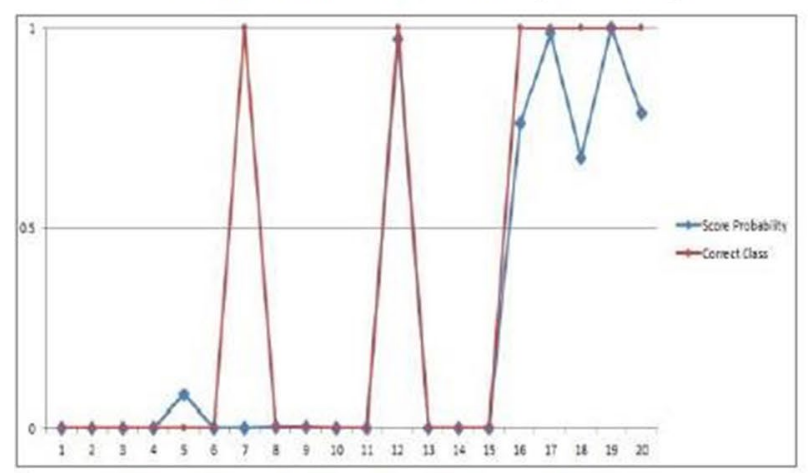

Score Probabilities for Antiwormal Activity

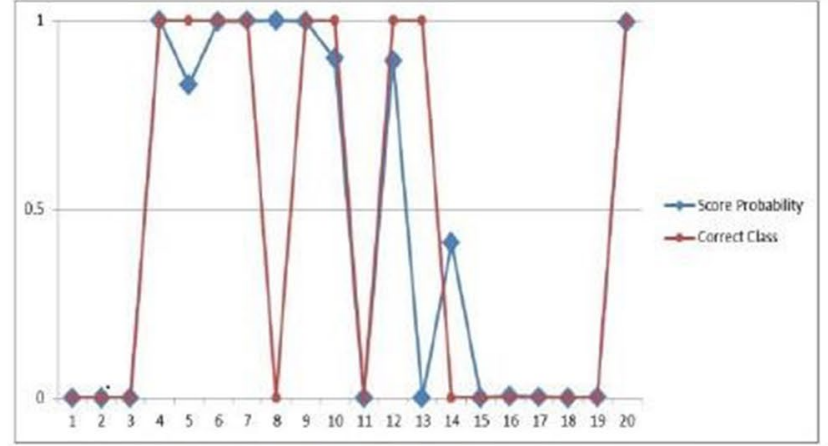

Score Probabilities for Antiviral Activity

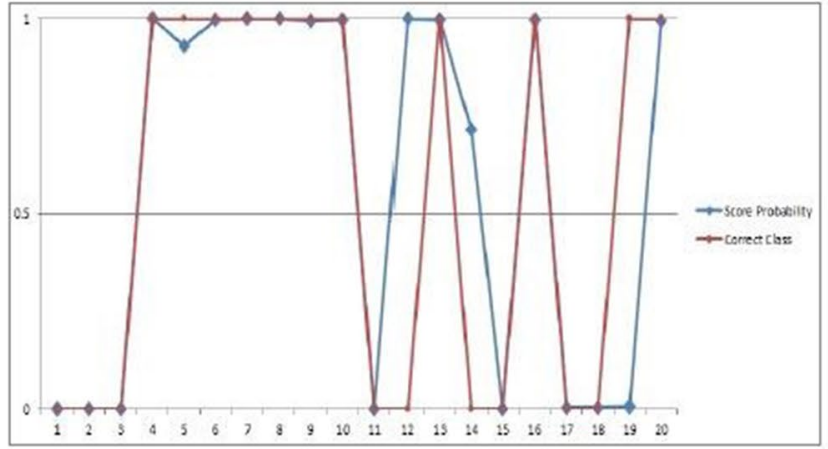

Score Probabilities for Antimicrobial Activity

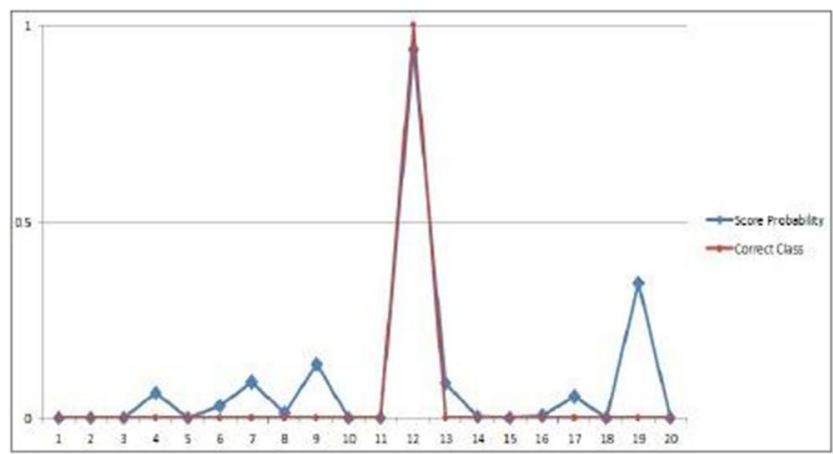

Score Probabilities for Anti-inflammatory Activity

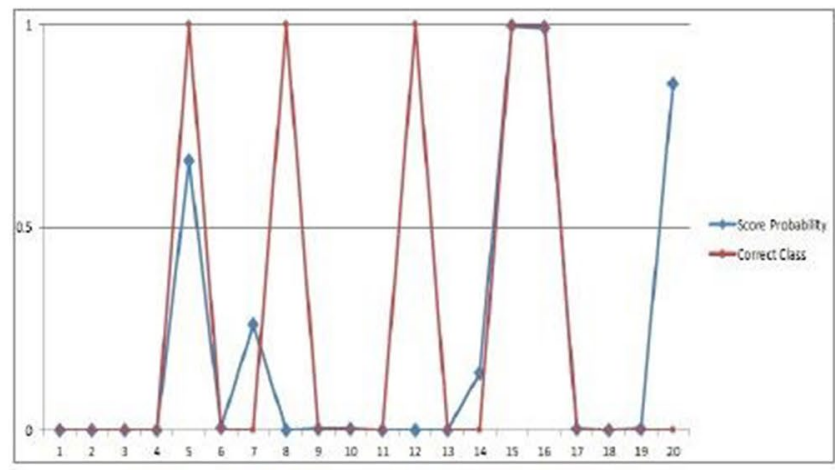

Score Probabilities for Cytotoxic Activity

Figure 2. The discrimination outcome of the developed CNN for predicting the biological activities for a new essential oils' dataset. 


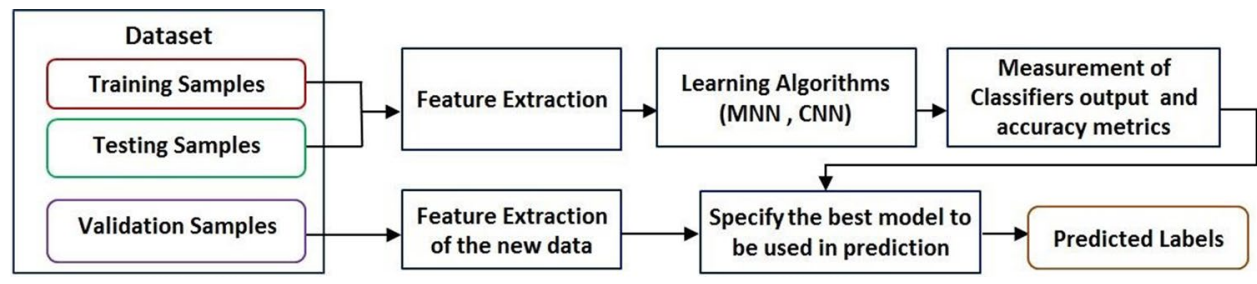

Figure 3. An inclusive flowchart for the proposed methodologies stages.

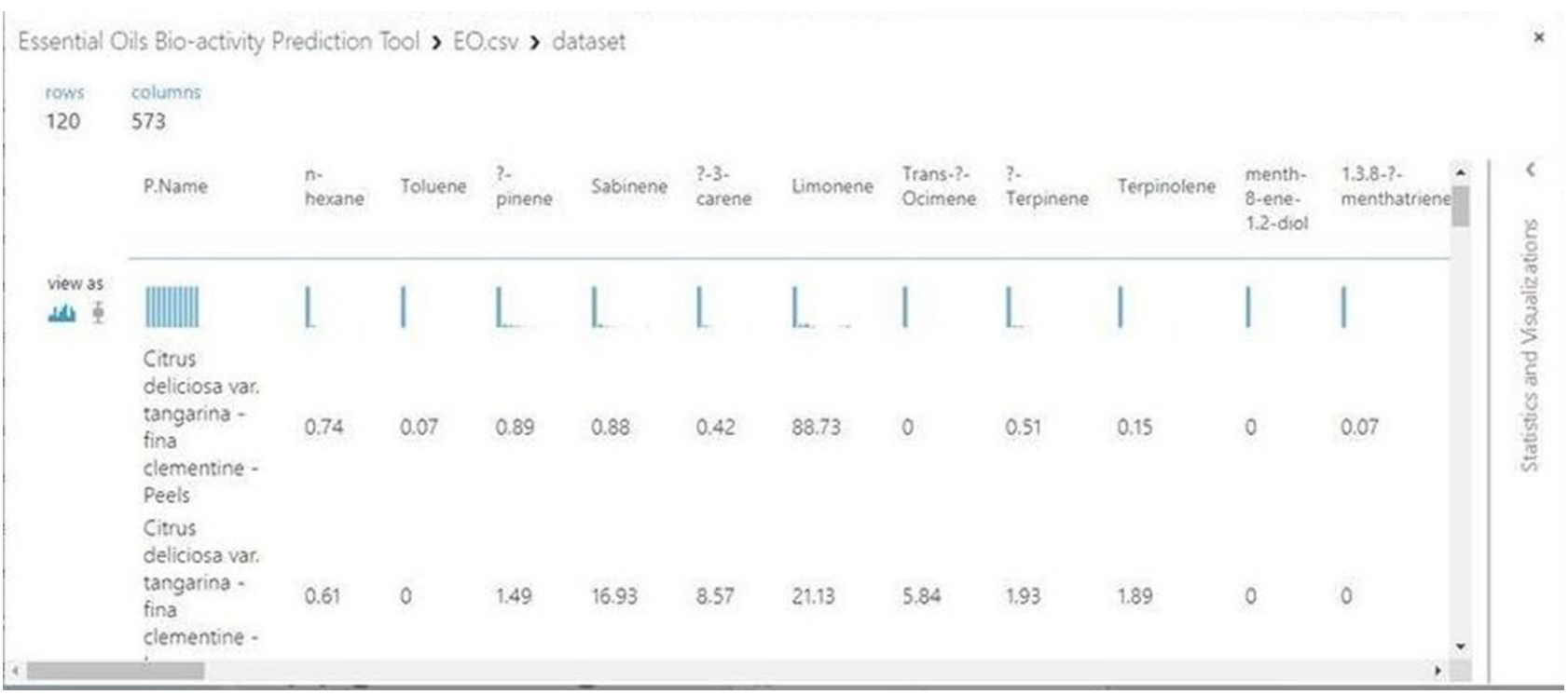

Figure 4. Sample for the Essential oils dataset imported by Azure. The Picture is taken from the Azure machine learning studio (classic) platform, https://studio.azureml.net/.

$$
\begin{gathered}
Z h_{j}=\sum_{j=1}^{n} x_{j} \cdot w_{j}+b \forall j=1, \ldots \ldots, n \\
O_{k}=\frac{e^{O h_{k}}}{\sum_{k=1}^{L} e^{O h_{k}}} \forall k=1 \ldots .8
\end{gathered}
$$

where

$$
O h_{k}=\sum_{i=1}^{50} o h_{i} \cdot w_{i}
$$

In general, MNN proves its efficiency when the total amount of data is limited. Hereby, more significantly, another efficient classification model based on the CNN is created. The proposed CNN is developed in such a way to handle the ambiguity and inconsistency that appeared in the chemical compositions values which could not be fully treated with the MNN. One of the mysterious characteristics in the essential oil(s) is that, they may contain some chemical compounds that have no biological influence activity, and this may be due to the inconsistency among these compounds. Thus, a number of compounds/oils that have no effect in a certain EO's pool may be found, but they may show a clear influence if they are found with other compounds in another EO pool ${ }^{58}$.

The problem in this study is categorized as multi-label classification problem, where the essential oils can have multiple activities (i.e. outputs) at the same time. Thus, in regards with the proposed dataset, the CNN could produce eight output labels for each essential oil (i.e. the eight biological activities), where an essential oil may have all of these activities or some of them. The output labels have been encoded in the form of a one-hot encoded vector with multiple ones in it, as a special form of the one-hot encoding method. For instance, the Essential oil "Pluchea dioscoridis" is known with its activities as antimicrobial, antioxidant, and anticancer, so its label will be $[0,0,0,1,1,1,0,0]$ for the target vector [Antiviral, Antiwormal, Anti-inflammatory, Anticancer, Antioxidant, Antimicrobial, Antifungal, and Cytotoxic Activity]. Table 5 shows a sample of the CNN training results on the dataset where it documents the score probabilities that outcome from the sigmoid activation function. These score values refer to predicted biological activities labels for a number of Essential oils. 


\begin{tabular}{|c|c|c|c|c|c|c|c|c|}
\hline $\begin{array}{l}\text { Eos name } \\
\text { Score probabilities }\end{array}$ & Antiviral & Antiwormal & Anti-inflammatory & Anticancer & Antioxidant & Antimicrobial & Antifungal & Cytotoxic activity \\
\hline $\begin{array}{l}\text { Citrus deliciosa var. tangarina-fina clem- } \\
\text { entine-peels }\end{array}$ & 0.9888 & 0.0014 & 0.0032 & 0.0001 & 0.9854 & 0.9981 & 0.9983 & 0.9966 \\
\hline $\begin{array}{l}\text { Citrus deliciosa var. tangarina-fina clem- } \\
\text { entine-leaves }\end{array}$ & 0.9999 & 0.9986 & 0.0005 & 0.0003 & 0.9992 & 0.9984 & 0.9983 & 0.0013 \\
\hline $\begin{array}{l}\text { Citrus deliciosa var. tangarina-Nour } \\
\text { Clementine-peels }\end{array}$ & 0.9465 & 0.0022 & 0.3243 & 0.0002 & 0.9894 & 0.9854 & 0.9684 & 0.7711 \\
\hline $\begin{array}{l}\text { Citrus deliciosa var. tangarina-Nour } \\
\text { Clementine-leaves }\end{array}$ & 0.9999 & 0.0013 & 0.0641 & 0.0002 & 0.9995 & 0.9997 & 0.9996 & 0.0003 \\
\hline $\begin{array}{l}\text { Citrus deliciosa var. tangarina-Spinosa } \\
\text { Clementine-peels }\end{array}$ & 0.8294 & 0.0795 & 0.0001 & 0.0003 & 0.8291 & 0.9294 & 0.9993 & 0.6654 \\
\hline $\begin{array}{l}\text { Citrus deliciosa var. tangarina-Spinosa } \\
\text { Clementine-leaves }\end{array}$ & 0.9977 & 0.0015 & 0.0320 & 0.0084 & 0.9968 & 0.9982 & 0.9961 & 0.0049 \\
\hline $\begin{array}{l}\text { Citrus deliciosa var. tangarina-Thornless } \\
\text { Clementine-peels }\end{array}$ & 0.9991 & 0.0007 & 0.0931 & 0.0034 & 0.9987 & 0.9999 & 0.998 & 0.2611 \\
\hline $\begin{array}{l}\text { Citrus deliciosa var. tangarina-Thornless } \\
\text { Clementine-leaves }\end{array}$ & 0.9999 & 0.0020 & 0.0134 & 0.0082 & 0.9999 & 0.9998 & 0.9996 & 0.0007 \\
\hline Callistemon comboynensis & 0.9975 & 0.0037 & 0.1375 & 0.0011 & 0.9947 & 0.9955 & 0.9945 & 0.0031 \\
\hline $\begin{array}{l}\text { Cupressus sempervirens } \mathrm{L} \\
\text { Semperuirens } \mathrm{L}\end{array}$ & 0.8982 & 0.0011 & 0.0004 & 0.0012 & 0.9947 & 0.9967 & 0.0002 & 0.0035 \\
\hline Cuminum cyminum & 0.8923 & 0.9747 & 0.9384 & 0.0005 & 0.9297 & 0.9997 & 0.7909 & 0.0001 \\
\hline Ocimum basilicum L. (sinai ) & 0.0004 & 0.0005 & 0.0883 & 0.9977 & 0.9991 & 0.9990 & 0.9089 & 0.0003 \\
\hline Tagetes minuta $\mathrm{L}$ & 0.4107 & 0.0011 & 0.0034 & 0.0253 & 0.5146 & 0.7167 & 0.6179 & 0.1418 \\
\hline Achillea fragrantissima & 0.0004 & 0.0003 & 0.0003 & 0.0007 & 0.0008 & 0.0013 & 0.0001 & 0.9985 \\
\hline Pluchea dioscoridis & 0.0052 & 0.7622 & 0.0054 & 0.9863 & 0.9931 & 0.9991 & 0.8932 & 0.9915 \\
\hline Myrtus communis-leaves & 0.0022 & 0.9887 & 0.0554 & 0.0018 & 0.0003 & 0.0021 & 0.0023 & 0.0028 \\
\hline Myrtus communis-fruits & 0.0007 & 0.6754 & 0.0001 & 0.0005 & 0.0013 & 0.0023 & 0.0011 & 0.0004 \\
\hline Eugenia supraxillaris-leaves & 0.001 & 0.9998 & 0.3451 & 0.0075 & 0.0050 & 0.0075 & 0.0065 & 0.0032 \\
\hline Eugenia supraxillaris-fruits & 0.9955 & 0.7865 & 0.0003 & 0.0003 & 0.9995 & 0.9934 & 0.0273 & 0.8539 \\
\hline
\end{tabular}

Table 5. A sample of the Score Probabilities for the CNN Training Process.

\section{Conclusion}

Developing a computational model based on deep learning for classifying and predicting the EOs' biological activities without resorting to in-vitro experiments is the challenge of this study.

Due to the efficacy of the EOs as antiseptics, antimicrobials, antifungals, antioxidant, antitumor, antivirals, and anti-inflammatories, they have a great attention from the health concerns industries, such as medicine, pharmaceutics, cosmetics, and others. However, there is a significant challenge in deciding the relevance between the chemical compounds that form the EO and its biological activities through the traditional in-vitro experiments. In this study, two classification models are implemented to classify and predict the biological activities of 120 types of Egyptian essential oils as an experimental study case. This experiment has been implemented based on two types of supervised learning algorithms, Multiclass Neural Network and Convolutional Neural Network in order to evaluate the efficiency of both machine and deep learning techniques. The comparison between the accuracy and relevance metrics for both $\mathrm{MNN}$ and $\mathrm{CNN}$ algorithms in the testing stage showed that the CNN outperformed the MNN as it scored an accuracy rate $98.13 \%$, while the MNN recorded $81.88 \%$.

\section{Method}

The model of the CNN, which is utilized in this experiment, comprises of a three-layers fully connected network with two convolution layers and two pooling layers followed by one hidden layer as shown in Fig. 5. The input information for the proposed $\mathrm{CNN}$ is a $2 \mathrm{D}$ matrix of $[120 \times 573]$ that represents "the number of essential oils and the numbers of their chemical compounds, respectively". The following sequential layers start with a convolution layer which applies a convolutional process to the input matrix. The output of each node in the convolution layer is the result of a convolution operation by each filter. The next layer is the pooling layer which is used to compress information and generalize features to reduce the overfitting of the training data. In this experiment, the local max-pooling, which produces the maximum value from small divided regions in the input matrix, is used. The convolution and pooling outputs are calculated by the following equation;

$$
O_{k}^{l}=\sum a_{k}^{l} * w_{k}^{l}+b_{k}^{l}
$$

where $O_{k}^{l}$ is the output vector of the $l$ th layer with $k$ th kernel, $a_{k}^{l}$ is the input vector, $w_{k}^{l}$ is the weight of the convolution/pooling filter, and $b_{k}^{l}$ is the bias coefficient.

During the CNN learning process, seven hyper-parameters are tuned (convolution filter size $(k)$, number of filters $(f)$, stride size $(s)$, pooling size $(p)$, number of nodes in the hidden layer, and the activation function). The 

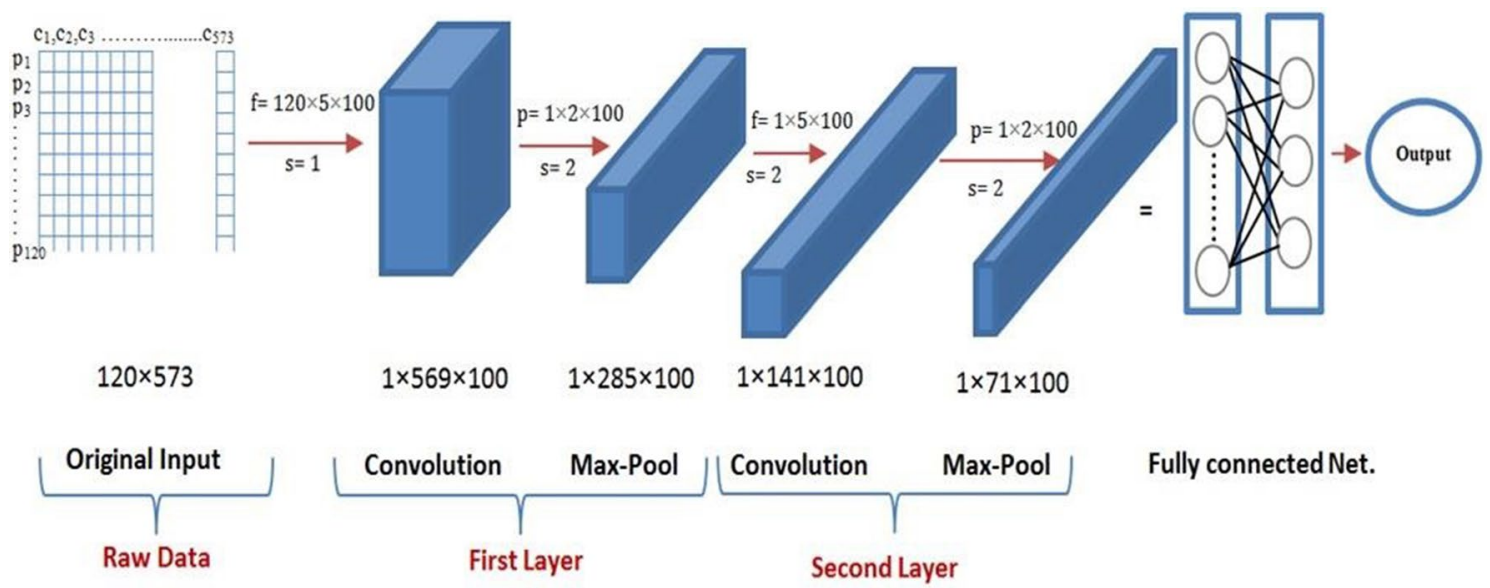

Figure 5. The Architecture of the Proposed CNN, it mainly begins with an original input matrix represents the essential oils data. The internal network schema based on two layers of convolution and pooling. The output of these layers feeds a fully connected feed forward neural network that uses a sigmoid activation function to find the appropriate output.

\begin{tabular}{|l|l|l|l|l|}
\hline Layers & Filter size & Stride & Output size & Output Dense \\
\hline Input & - & - & $120 \times 573$ & 68,760 \\
\hline 1st convolution layer & $120 \times 5 \times 100$ & 1 & $1 \times 569 \times 100$ & 56,900 \\
\hline 1st max pooling layer & $1 \times 2 \times 100$ & 2 & $1 \times 285 \times 100$ & 28,500 \\
\hline 2nd convolution layer & $1 \times 5 \times 100$ & 2 & $1 \times 141 \times 100$ & 14,100 \\
\hline 2nd max pooling layer & $1 \times 2 \times 100$ & 2 & $1 \times 71 \times 100$ & 7100 \\
\hline
\end{tabular}

Table 6. Description of CNN layers with the hyper-parameters values.

numbers of iterations for the learning process are specified around 100. Table 6 displays the values of the assumed hyper-parameters and the output layer dimensions. The individual layer dimension can be calculated by ${ }^{8}$;

$$
n^{l} \times m^{l}=\left[\left[\frac{n^{l-1}-f^{l}}{s^{l}}\right]+1\right] \times\left[\left[\frac{m^{l-1}-f^{l}}{s^{l}}\right]+1\right]
$$

Finally, the sequence of the convolutional and pooling layers ends with a fully connected feed-forward neural layer that uses a "sigmoid" activation function. The sigmoid function is selected in the proposed CNN implementation because its function depends mainly on converting each score of the final node to a probability value between 0 to 1 , independent of what the other scores are. So, the input could be classified into multiple independent classes (Suppl Information).

\section{Data availability}

The complete data set is available in the supplementary file (sup-file). The dataset is formatted to be suitable to be processed by Azure ML modules.

Received: 18 May 2020; Accepted: 20 November 2020

Published online: 07 December 2020

\section{References}

1. Muniz, I. et al. Classification of food vegetable oils by fluorimetry and artificial neural networks. Food Control 47, 86-91 (2015).

2. Littmann, M. et al. Validity of machine learning in biology and medicine increased through collaborations across fields of expertise. Mach. Intell. Nat. https://doi.org/10.1038/s42256-019-0139-8 (2016).

3. Dhifi, W., Bellili, S., Sabrine Jazi, N. B. W. M. Essential oils' chemical characterization and investigation of some biological activities. Medicines 3, 1-16 (2020).

4. Arjmand, Z. \& Dastan, D. Chemical characterization and biological activity of essential oils from the aerial part and root of Ferula haussknechtii. Flavour Fragr. 114-123. https://doi.org/10.1002/ff.3544 (2020).

5. Harper, G., Bradshaw, J., Gittins, J. C., Green, D. V. S. \& Leach, A. R. Prediction of biological activity for high-throughput screening using binary kernel discrimination. J. Chem. Inf. Comput. Sci. 41, 1295-1300 (2001).

6. Jones, D. T. Setting the standards for machine learning in biology. Nat. Rev. Mol. Cell Biol. 20, 659-660 (2019).

7. Aoki, G. \& Sakakibara, Y. Convolutional neural networks for classification of alignments of non-coding RNA sequences. Bioinformatics. https://doi.org/10.1093/bioinformatics/bty228 (2018).

8. Dhillon, A. \& Verma, G. K. Convolutional neural network: A review of models, methodologies and applications to object detection. Prog. Artif. Intell. https://doi.org/10.1007/s13748-019-00203-0 (2019). 
9. Gao, R. X. et al. Deep learning for smart manufacturing : Methods and applications Deep learning for smart manufacturing : Methods and applications. J. Manuf. Syst. 48, 144-156 (2018).

10. Tharwat, A. Applied computing and informatics classification assessment methods. Appl. Comput. Inform. 1-13. https://doi. org/10.1016/j.aci.2018.08.003 (2018).

11. Elansary, H. O., Abdelgaleil, S. A. M., Mahmoud, E. A., Yessoufou, K. \& Elhindi, K. Effective antioxidant, antimicrobial and anticancer activities of essential oils of horticultural aromatic crops in northern Egypt. 1-10 (2018).

12. Ragno, R. et al. Essential oils against bacterial isolates from cystic fibrosis patients by means of antimicrobial and unsupervised machine learning approaches. Sci. Reports Nat. Res. 10, 1-11 (2020).

13. Artini, M., Vrenna, G. \& Pepi, F. Antimicrobial and antibiofilm activity and machine learning classification analysis of essential oils. Molecules 23 (2018).

14. Fayez, S., Abouziena, H. F., El-razik, T. M. A., Amer, H. M. \& Hussein, M. S. Winter weeds and its control in the medicinal plants in Egypt : A survey study. Egypt. Pharm. J. 18, 16-26 (2019).

15. El-askary, H. I. et al. Chemical composition of the essential oil and botanical study of the flowers of Mentha suaveolens chemical composition of the essential oil and botanical study. Inf. Healthc. 52, 688-697 (2014).

16. Kassem, F. F., Harraz, F. M. \& Material, P. Composition of the essential oil of Egyptian Cupressus L. cones. Flavour Fragr. J. 6, 205-207 (1991).

17. El-azim, M. H. M. A., Abdelgawad, A. A. M. \& El-mesallamy, A. M. D. Chemical composition and antimicrobial activity of essential oil of Egyptian Ocimum basilicum L indo. Am. J. Pharm. Sci. 2, 837-842 (2015).

18. Aziz, E. E., Badawy, E. M., Zheljazkov, V. D., Nicola, S. M. \& Fouad, H. Yield and chemical composition of essential oil of Egypt. J. Chem. 62, 533-540 (2019).

19. Aziz, E. E. et al. Chemical composition of Mentha pulegium L. (Pennyroyal) plant as influenced by foliar application of different sources of zinc. Egypt. Pharm. J. 18, 53-59 (2019).

20. Khalil, N., Ashour, M., Fikry, S., Naser, A. \& Salama, O. Chemical composition and antimicrobial activity of essential oils of selected Apiaceous plants growing in Egypt Future. Futur. J. Pharm. Sci. 4, 88-92 (2018).

21. El-hawary, S. S. et al. Chemical composition and biological activities of peels and leaves essential oils of four cultivars of Citrus deliciosa var. tangarina. Am. J. Essent. Oils Nat. Prod. 1, 1-6 (2013).

22. El, A., El, N. \& Abd-elgawad, A. M. Essential oils constituents of aerial parts of Cyperus capitatus L. and Cyperus difformis L. grown wild in Egypt. J. Essent. Oil Bear. Plants 20, 1659-1665 (2017).

23. Abd-elgawad, A. M. et al. Chemical composition variations, allelopathic, and antioxidant activities of Symphyotrichum squamatum (Spreng.) Nesom essential oils growing in heterogeneous habitats. Arab. J. Chem. https://doi.org/10.1016/j.arabjc.2019.07.005 (2019).

24. Amin, S. M. et al. Comparative chemical study and antimicrobial activity of essential oils of three Artemisia species from Egypt and Saudi Arabia. Wiley Online Libr. Flavour Fragr. J. 34, 450-459 (2019).

25. Saqqa, G. Al, Alian, A., Ismail, F. \& Ramzy, S. Chemical composition of rocket, thyme and parsley essential oils and their effect on some fungi and aflatoxin production. MedCrave Online J. Toxicol. 4, 277-282 (2018).

26. Ahl, H. A. H. S., Gendy, A. S. H., Mahmoud, A. A. \& Mohamed, H. F. Y. Essential oil composition of Marrubium vulgare L. cultivated in Egypt. Int. J. Plant Sci. Ecol. 1, 138-141 (2015).

27. Tarek, N. et al. ScienceDirect comparative chemical and antimicrobial study of nine essential oils obtained from medicinal plants growing in Egypt. Beni-Suef Univ. J. Basic Appl. Sci. 3, 149-156 (2014).

28. Ghareeb, M. A., Refahy, L. A., Saad, A. M. \& Ahmed, W. S. CODEN (USA): PCHHAX chemical composition, antioxidant and anticancer activities of the essential oil from Eucalyptus citriodora (Hook.) leaves. Der Pharma Chem. 8, 192-200 (2016).

29. El-ghorab, A. H., Ramadan, M. M., El-moez, S. I. A., April, M. \& April, M. Research journal of pharmaceutical, biological and chemical sciences essential oil, antioxidant, antimicrobial and anticancer activities of Egyptian Pluchea dioscoridis extract. Res. J. Pharm. Biol. Chem. Sci. 6, 1255-1265 (2015).

30. Halim, Ahmed F; Eldahshan, O. Comparison of the composition and antimicrobial activities of the essential oils of green branches and leaves of Egyptian navel orange (Citrus sinensis L.) O Sbeck. Chem. Biodivers. 13, 681-685 (2016).

31. Ohamed, M. I. E. M. \& Bdelgaleil, S. A. M. A. Chemical composition and insecticidal potential of essential oils from Egyptian plants against Sitophilus oryzae (L .) (Coleoptera : Curculionidae) and Tribolium castaneum (Herbst) (Coleoptera : Tenebrionidae). Appl. Entomol. Zool. 43, 599-607 (2008).

32. El Gohary, A. E., Hassan, E. A. \& Ali, A. F. Enhancement of growth, yield and chemical constituents of rosemary (Rosmarinus officinalis , L.) plants by application of compost and biofertilization treatments. Middle East J. Agric. Res. 4, 99-111 (2015).

33. Saad, M. M. G. Chemical composition and biological activities of four citrus essential oils. J. Plant Protein Path. 4, 767-780 (2013).

34. Aboutabl, E. A., El-tantawy, M. E. \& Shams, M. M. Chemical composition and antimicrobial activity of volatile constituents from the roots , leaves, and seeds of Arctium lappa L . (Asteraceae) grown in Egypt. Div. Pharm. Drug Ind. Res. Natl. Res. Center 12, 173-176 (2013).

35. Soliman, F. M., Yousif, M. F., Zaghloul, S. S. \& Okba, M. M. Seasonal variation in the essential oil composition of Origanum majorana L. cultivated in Egypt. Z Naturforsch C J. Biosci 64, 611-615 (2009).

36. Gad, H. A., Id, I. M. A. \& Wink, M. Phytochemical profiling and seasonal variation of essential oils of three Callistemon species cultivated in Egypt. PLoS ONE 17, 1-15 (2019).

37. Kouachea, B. et al. Chemical composition and acaricidal activity of Thymus algeriensis essential oil against Varroa destructor. NPC Nat. Prod. Commun. 12, 3135-3138 (2017).

38. Ibrahim, M. E., Ahmed, S. S., E.-S. \& S. A. Essential oil composition of Iphiona scabra (DC) grow wild in Egypt. Int. Food Res. J. 23, 1823-1826 (2016).

39. Aboutabl, E. A., Meselhy, K. M., Elkhreisy, E. M., Nassar, M. I. \& Fawzi, R. Journal of essential oil bearing plants composition and bioactivity of essential oils from leaves and fruits of Myrtus communis and Eugenia supraxillaris (Myrtaceae) grown in Egypt. J. Essent. Oil Bear. Plants 14, 37-41 (2013).

40. Hamdan, D. I., Abdulla, R. H., Mohamed, M. E. \& El-shazly, A. M. Chemical composition and biological activity of essential oils of Cleopatra mandarin (Citrus reshni) cultivated in Egypt. J. Pharmacogn. Phyther. 5, 83-90 (2013).

41. El-Kholany, E. Utilization of essential oils from Citronella and Geranium as natural preservative in mayonnaise Ebtehal. Int. J. Microbiol. Biotechnol. 1, 49-59 (2016).

42. El-kashoury, E. A., El-askary, H. I. \& Kandil, Z. A. Chemical composition and biological activities of the essential oil of Mentha suaveolens Ehrh. Z. Naturforsch. C. J. Biosci. 67, 571-579 (2012).

43. Rowayshed, G. H. et al. Effective chemical compounds and antibacterial activities of marjoram leaves, teucrium leaves and fennel fruits essential oils. Middle East J. Appl. Sci. 4, 637-647 (2014).

44. Vanessa, R. et al. Chemical composition and antifungal activity of essential oils from four Asteraceae plants grown in Egypt. $Z$. Naturforsch C J. Biosci. 73, 313-318 (2018).

45. El-Shazly, A., Hafez, S. \& Wink, M. Comparative study of the essential oils and extracts of Achillea fragrantissima (Forssk.) Sch. Bip. and Achillea santolina L. (Asteraceae) from Egypt. Pharmazie 59, 226-230 (2004).

46. Kf, T. \& Zta, S. Chemical composition and antibacterial activity of volatile oil of Sequoia sempervirens (Lamb.) grown in Egypt medicinal \& aromatic plants. Med. Aromatic Plants 5 (2016). 
47. Soliman, F. M., Fathy, M. M., Salama, M. M. \& Saber, F. R. Chemical composition and bioactivity of the volatile oil from leaves and stems of Eucalyptus cinerea. Inf. Healthc. 52, 1272-1277 (2014).

48. Ramadan, M. M., Ali, M. M., Ghanem, K. Z. \& El-Ghorabe, A. H. Essential oils from Egyptian aromatic plants as antioxidant and novel anticancer agents in human cancer cell lines. Grasas y Aceites 66 (2015).

49. Mohamed, A. A. et al. Assessment of antioxidant and antimicrobial activities of essential oil and extracts of Boswellia carteri resin. Int. J. Pharmacogn. Phytochem. Res. 7, 502-509 (2015).

50. Ibrahim, T. A., El-hela, A. A., El-hefnawy, H. M. \& Al-taweel, A. M. Chemical composition and antimicrobial activities of essential oils of some coniferous plants cultivated in Egypt. Iran. J. Pharm. Res. 16, 328-337 (2017)

51. Fayed, S. A. Antioxidant and anticancer activities of Citrus reticulate (Petitgrain Mandarin) and Pelargonium graveolens (Geranium) essential oils. Res. J. Agric. Biol. Sci. 5, 740-747 (2009).

52. Hanafi, R. S., Sobeh, M., Ashour, M. L. \& El-readi, M. Z. Chemical composition and biological activity of essential oils of cumin and coriander fruits from Egypt chemical composition and biological activity of essential oils of cumin and coriander fruits from Egypt. Nat. Prod. J. 4, 63-69 (2014).

53. Abdelhady, M. I. \& Aly, H. A. H. Original article antioxidant and antimicrobial activities of Callistemon comboynensis essential oil. Free Radic. Antioxid. 2, 37-41 (2012).

54. Mahmoud, G. I. Biological effects, antioxidant and anticancer activities of marigold and basil essential oils. J. Med. Plants Res. 7, 561-572 (2013).

55. Badawy, M. E. I. \& Abdelgaleil, S. A. M. Composition and antimicrobial activity of essential oils isolated from Egyptian plants against plant pathogenic bacteria and fungi. Ind. Crop. Prod. 52, 776-782 (2014).

56. https://studio.azureml.net/. https://doi.org/10.1109/ICCES.2016.7821969

57. Wang, Y., Li, Y., Song, Y. \& Rong, X. Applied sciences the influence of the activation function in a convolution neural network model of facial expression recognition. Appl. Sci. 10 (2020).

58. Daynac, M., Cortes-cabrera, A. \& Prieto, J. M. Application of artificial intelligence to the prediction of the antimicrobial activity of essential oils. Evidence-Based Complement. Altern. Med. 2015 (2015).

\section{Acknowledgements}

This project was supported financially by the Academy of Scientific Research and Technology (ASRT), Egypt, Grant No. 6474.

\section{Author contributions}

N.E.E. and W.A.A. designed and carried out the experiments and developed the machine and deep learning algorithms. M.K.H. and O.A. revised and adopted the dataset. All authors contributed in writing, editing, and revising the manuscript.

\section{Additional information}

Supplementary information is available for this paper at https://doi.org/10.1038/s41598-020-78449-1.

Correspondence and requests for materials should be addressed to N.E.E.-A.

Reprints and permissions information is available at www.nature.com/reprints.

Publisher's note Springer Nature remains neutral with regard to jurisdictional claims in published maps and institutional affiliations.

(c) (i) Open Access This article is licensed under a Creative Commons Attribution 4.0 International License, which permits use, sharing, adaptation, distribution and reproduction in any medium or format, as long as you give appropriate credit to the original author(s) and the source, provide a link to the Creative Commons licence, and indicate if changes were made. The images or other third party material in this article are included in the article's Creative Commons licence, unless indicated otherwise in a credit line to the material. If material is not included in the article's Creative Commons licence and your intended use is not permitted by statutory regulation or exceeds the permitted use, you will need to obtain permission directly from the copyright holder. To view a copy of this licence, visit http://creativecommons.org/licenses/by/4.0/.

(C) The Author(s) 2020 